\title{
Variables individuales y escolares en estudiantes universitarios víctimas de ciberacoso
}

\author{
Individual and School Variables in University Students Victims of Cyberbullying
}

Variáveis individuais e escolares em estudantes universitários vítimas de cyberbullying

Esperanza Vargas Jiménez

Universidad de Guadalajara, México

esperanzavgas@hotmail.com https://orcid.org/0000-0002-6943-2878

Remberto Castro Castañeda

Universidad de Guadalajara, México reembert@hotmail.com https://orcid.org/0000-0002-5916-7839

Claudia Gregoria Huerta Zúñiga

Universidad de Guadalajara, México claughz_@hotmail.com https://orcid.org/0000-0002-5735-8068

Raquel Domínguez Mora Universidad de Guadalajara, México raqueldmz@gmail.com https://orcid.org/0000-0002-9382-3174

Raúl Medina Centeno

Universidad de Guadalajara, México topraul2002@yahoo.co.uk https://orcid.org/0000-0002-9277-5561 


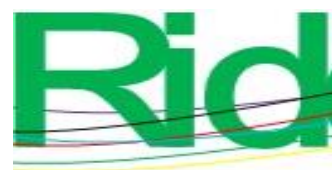

\section{Resumen}

El uso de las tecnologías de la información y de la comunicación conlleva beneficios y perjuicios. Actualmente uno de los riesgos que generan mayor preocupación es el fenómeno del acoso en las redes sociales. A nivel universitario existen pocos estudios que expliquen el impacto individual y escolar del acoso por Internet en las víctimas. Considerando estos antecedentes, el objetivo principal de este estudio fue examinar la cibervictimización y su relación con variables individuales (aceptación percibida y depresión) y escolares (implicación, afiliación y ayuda del profesor), analizando las diferencias en función del sexo, examinando las divergencias entre los grupos que documentaron cibervictimización severa, ocasional y nula, y evidenciando el valor predictivo de las variables individuales y escolares de este fenómeno. Esta investigación fue de tipo explicativa y se utilizó un diseño transversal. La muestra fue representativa, conformada por 662 jóvenes universitarios, 282 hombres (42.5\%) y 380 mujeres (57.5\%), con un rango de edad entre los 18 y 25 años y una media de 19.41 años (DT = 2.43). Se realizó un análisis de correlaciones de Pearson, y se obtuvieron correlaciones significativas entre todas las variables. La prueba $t$ de Student reveló diferencias estadísticamente significativas entre los sexos: los chicos obtuvieron medias más altas en aceptación percibida de la familia, la madre y el padre, así como puntuaciones elevadas en implicación escolar y ayuda del profesor; en cambio, las chicas sobresalen en sintomatología depresiva. En el análisis de varianza se establecieron tres grupos de contraste: cibervíctimas severas, cibervíctimas ocasionales y no cibervíctimas, comparando las variables individuales y escolares; las no cibervíctimas obtuvieron las puntuaciones más elevadas, en comparación con los jóvenes con moderada y severa cibervictimización, en afiliación escolar y aceptación percibida de los amigos, la familia, la madre y el padre. En la variable implicación escolar y ayuda del profesor no hay diferencias en las medias de los grupos de no cibervíctimas y ocasionales cibervíctimas, pero sí de ambos grupos con respecto a las víctimas severas. Además, los adolescentes con severa cibervictimización mostraron una mayor sintomatología depresiva que los jóvenes con moderada y no cibervictimización. Los análisis de regresión confirmaron el valor predictivo de las variables individuales (11.4\%) y escolares (7.0\%) en la cibervictimización. Se confirmó el impacto negativo de las agresiones a través de las redes sociales en la salud mental y en la identidad de las víctimas sin distinción de edad o grado académico. Aunado a ello, se corrobora que el ciberacoso es un fenómeno que debe abordarse sistémicamente, involucrando 


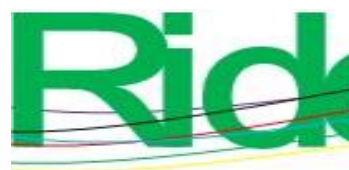

Revista Iberoamericana para la

Investigación y el Desarrollo Educativo

ISSN 2007 - 7467

aspectos individuales, escolares, familiares y sociales, haciendo énfasis en la importancia de la colaboración entre la familia, las autoridades escolares y profesorado.

Palabras clave: aceptación percibida, afiliación, ayuda del profesor, cibervictimización, depresión, implicación.

\section{Abstract}

The use of information and communication technologies entails benefits and damages. Currently one of the risks that generate most concern is the phenomenon of bullying through social media. At the college level there are few studies that explain the individual and school impact bullying through Internet on victims. Considering this background, the main objective of this study was to examine cybervictimization and its relationship with individual (perceived acceptance and depression) and school variables (involvement, affiliation and teacher aid), analyzing the differences according to sex, examining the differences between the groups that reported severe, occasional and zero cybervictimization, and evidencing the predictive value of the individual and school variables of this phenomenon. This research was explanatory and a cross-sectional design was used. The sample was representative, consisting of 662 young university students, 282 men (42.5\%) and 380 women (57.5\%), with an age range between 18 and 25 years and an average of 19.41 years $(\mathrm{SD}=2.43)$. An analysis of Pearson's correlations was performed, obtaining significant correlations between all the variables. The Student's $t$ test revealed statistically significant differences between the sexes: the boys obtained higher means in perceived acceptance of the family, the mother and the father, as well as high scores in school involvement and teacher support; however, the girls stand out in symptomatology depressive. In the analysis of variance three contrast groups were established: severe cyber victims, occasional cyber victims and noncyber victims comparing the individual and school variables; non-cyber victims obtained the highest scores, compared to young people with moderate and severe cybervictimization, in school affiliation and perceived acceptance of friends, family, mother and father. In school involvement and teacher support variables there are no differences in the means of the non-cyber-victim and occasional cyber-victim groups, but there's difference of both groups with respect to the severe victims. In addition, adolescents with severe cybervictimization have a higher depressive symptomatology than young people with moderate and do not have cybervictimization. Regression analyzes confirmed the predictive value of individual (11.4\%) and school (7.0\%) variables in 


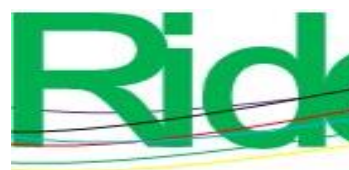

Revista Iberoamericana para la Investigación y el Desarrollo Educativo

ISSN 2007 - 7467

cybervictimization. The negative impact of the aggressions through social networks on mental health and on the identity of the victims was confirmed regardless of age or academic degree. In addition to this, it is corroborated that cyberbullying is a phenomenon that must be addressed systemically, involving individual, school, family and social aspects, and emphasizing the importance of collaboration between the family, school authorities and teachers.

Keywords: perceived acceptance, affiliation, teacher aid, cybervictimization, depression, involvement.

\section{Resumo}

O uso de tecnologias da informação e comunicação acarreta benefícios e danos. Atualmente, um dos riscos que mais preocupa é o fenômeno do assédio nas redes sociais. No nível universitário, existem poucos estudos que explicam o impacto individual e escolar do assédio na Internet nas vítimas. Considerando esse cenário, o principal objetivo deste estudo foi examinar a cibervictimização e sua relação com variáveis individuais (percepção de aceitação e depressão) e escolares (envolvimento, afiliação e apoio ao professor), analisando diferenças com base no sexo, examinando diferenças entre os grupos que documentaram cibervitimização grave, ocasional e nula e evidenciaram o valor preditivo das variáveis individuais e escolares desse fenômeno. Esta pesquisa foi explicativa e foi utilizado um desenho transversal. A amostra foi representativa, composta por 662 jovens universitários, 282 homens (42,5\%) e 380 mulheres (57,5\%), com faixa etária entre 18 e 25 anos e média de 19,41 anos (DP =2,43). Foi realizada uma análise de correlação de Pearson, e correlações significativas foram obtidas entre todas as variáveis. O teste t de Student revelou diferenças estatisticamente significantes entre os sexos: os meninos obtiveram médias mais altas na aceitação percebida da família, mãe e pai, além de altas pontuações no envolvimento escolar e no apoio ao professor; em vez disso, as meninas se destacam na sintomatologia depressiva. Na análise de variância, três grupos de contraste foram estabelecidos: vítimas cibernéticas graves, vítimas ocasionais e não cibernéticas, comparando variáveis individuais e escolares; As vítimas não cibernéticas obtiveram as pontuações mais altas, comparadas aos jovens com cibervictimização moderada e grave, na afiliação à escola e na aceitação percebida de amigos, familiares, mãe e pai. Na variável envolvimento escolar e apoio ao professor, não há diferenças nos meios dos grupos de não-vítimas cibernéticas e ocasionais, mas de ambos os grupos em relação às vítimas graves. Além disso, os adolescentes com 


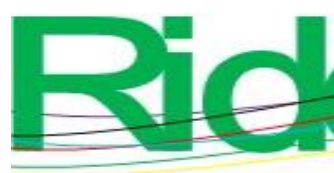

Revista Iberoamericana para la Investigación y el Desarrollo Educativo

ISSN 2007 - 7467

cibervictimização grave apresentaram sintomas depressivos maiores do que aqueles com moderação e não cibervictimização. As análises de regressão confirmaram o valor preditivo das variáveis individuais $(11,4 \%)$ e escolares $(7,0 \%)$ na cibervictimização. O impacto negativo das agressões pelas redes sociais na saúde mental e na identidade das vítimas foi confirmado sem distinção de idade ou grau acadêmico. Além disso, corrobora-se que o cyberbullying é um fenômeno que deve ser abordado sistemicamente, envolvendo aspectos individuais, escolares, familiares e sociais, enfatizando a importância da colaboração entre família, autoridades escolares e professores.

Palavras-chave: aceitação percebida, afiliação, ajuda do professor, ciber-vitimização, depressão, envolvimento.

Fecha Recepción: Septiembre 2019

Fecha Aceptación: Diciembre 2019

\section{Introducción}

El uso de las tecnologías de la información y de la comunicación (TIC) ha revolucionado las formas de convivencia, socialización, comunicación y de inclusión social (Buelga y Chóliz, 2013). Para los nativos digitales las pantallas forman parte de su vida cotidiana, lo que propicia la adquisición de habilidades tecnológicas; el ciberespacio es una realidad virtual creada por las computadoras, servidores y redes digitales que es contiguo al mundo real. En su formación universitaria, los jóvenes utilizan las TIC debido a que cubren necesidades educativas, de investigación y sociales. Sin embargo, las ventajas del empoderamiento tecnológico también integran riesgos y daños propiciados por el uso inadecuado, lo que genera niveles de toxicidad en la interacción comunicativa, poca capacidad empática con los otros (Udris, 2014) y la adaptación de diferentes identidades en cada situación online (Arab y Díaz, 2015). En el mundo virtual surgen fenómenos como el ciberacoso, en donde los jóvenes agreden a compañeros de clase sin considerar las consecuencias negativas de este fenómeno en la personalidad de la cibervíctima: su autopercepción, su estado de ánimo y las consecuencias en las redes de apoyo.

El ciberacoso se entiende como el fenómeno en donde se intimida y maltrata a coetáneos a través del Internet, la computadora, el teléfono celular, los videojuegos online y las TIC (Buelga, Cava y Musitu, 2012; Kowalski, Morgan y Limber, 2012; Patchin y Hinduja, 2015), con la intención de molestar o dañar a la víctima mediante la repetición de conductas agresivas y la permanencia de contenidos en las redes sociales (Gálvez, Vera, Cerda y Díaz, 2016; Garaigordobil 


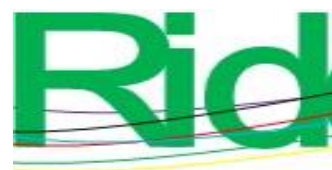

Revista Iberoamericana para la Investigación y el Desarrollo Educativo

ISSN 2007 - 7467

y Martínez, 2016). Se puntualiza en conductas como insultos, denigración, hostigamiento, suplantación de la identidad, exclusión, violación de la intimidad y persecución, ya sea de forma directa o indirecta (Kowalski, Giumetti, Schroeder y Lattanner, 2014; Kowalski, Limber y Agatston, 2010), lo que genera un desequilibrio de poder entre el ciberacosador y la cibervíctima (Monelos, Mendiri y García-Fuentes, 2015) y crea un sentimiento de vulnerabilidad e indefensión por el anonimato del agresor (Lucas, Pérez y Giménez, 2016). A esto se suma la amplia audiencia de las redes sociales: las agresiones impactan en las dimensiones privadas y públicas por la gran cantidad de espectadores, lo que propicia un efecto devastador en la víctima por la falta de control de la situación (Ortega, Buelga y Cava, 2016), la rápida difusión de la información a una multitud de personas por el canal abierto del mundo virtual (Stewart, Drescher, Maack, Ebesutani y Young, 2014) y por el sentimiento de no poder escapar de la situación por parte de la cibervíctima (Durán y Martínez, 2015).

Para el entendimiento de la violencia entre iguales mediante el uso de las tecnologías, debemos partir de un fenómeno complejo e interconectado entre los recursos personales, los factores familiares, el clima escolar de la universidad y los factores sociales (Cross et al., 2015), por lo que el presente estudio retomó el enfoque ecológico y se centró en los factores individuales y escolares de los estudiantes universitarios.

Respecto a los factores individuales, la sintomatología depresiva se articula a la cibervictimización, en el sentido de la vulnerabilidad que perciben los grupos de iguales hacia la víctima (Cappadocia, Craig y Pepler, 2013); a su vez, la exposición a la violencia y al maltrato propicia una baja autoestima y mayor sintomatología depresiva (Gualdo, Hunter, Durkin, Arnaiz y Maquilón, 2015; Ortega, Torralba y Buelga, 2017; Resett y Putallaz, 2018). La sintomatología depresiva se caracteriza por una autopercepción negativa, sin disfrutar de la vida, con una tristeza arraigada al ser, con problemas de concentración y de sueño. Esta autorreferencia negativa de juicios de la cibervíctima mantiene y exagera la sintomatología depresiva (Lozano et al., 2016).

Otro factor individual estudiado es la aceptación percibida de las fuentes de apoyo social. El joven universitario en su red percibe aprobación, reconocimiento, calidez emocional, seguridad, comprensión y cuidado de parte de la madre, el padre, de la familia en general y los amigos. La familia y la red de amigos son apoyos inmediatos para los jóvenes. Ambos propician un clima cálido, una comunicación fluida y un reconocimiento, elementos que se relacionan directamente en la implicación del ciberacoso (Tanrikulu y Campbell, 2015). La percepción negativa de las fuentes de apoyo en la familia se caracteriza por baja comunicación y conflictos con los padres 


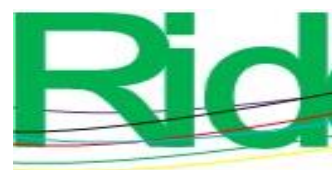

Revista Iberoamericana para la Investigación y el Desarrollo Educativo ISSN 2007 - 7467

(Buelga, Martínez y Musitu, 2016; Larrañaga, Yubero, Ovejero y Navarro, 2016; Romero et al., 2019), y posiciona a los jóvenes en un estado de vulnerabilidad y falta de recursos psicológicos protectores (Solecki, McLaughlin y Goldschmidt,2014). Así, un estilo parental autoritario basado en reglas rígidas que no cambian o se flexibilizan en la adolescencia tardía construye jóvenes inseguros, vulnerables y candidatos a convertirse en víctimas (Makri y Karagianni, 2014; Martínez, Murgui, García y García, 2019).

En la universidad, los alumnos y profesores suman un conjunto de percepciones subjetivas de las interacciones sociales y del contexto escolar que integran el clima escolar (Trickett, Leone, Fink y Braaten, 1993). El clima escolar cálido caracterizado por una sana convivencia, cooperación y empatía representa un factor protector del ciberacoso: en el aula los jóvenes muestran interés, atención y participan en clases, se construyen vínculos afectivos positivos, en donde los alumnos se conocen, conforman redes de amistades, lo que facilita el trabajo cooperativo en los proyectos, aunado al interés de los profesores, así como su preocupación y confianza en los alumnos, y un mayor tiempo de atención. Cuando el clima escolar es negativo, los jóvenes llevan a cabo conductas de acoso escolar, las cuales se trasladan al mundo virtual por el uso de las nuevas tecnologías (Kowalski et al., 2014); las cibervíctimas disminuyen su rendimiento académico (Giménez, Maquilón y Sánchez, 2014), fenómeno acompañado por la falta de concentración, ausentismo (Gualdo et al., 2015; Ortega et al., 2016), deterioro del autoestima y la autoconfianza, a lo que se le suman dificultades relacionales con sus compañeros (Navarro, Ruiz, Larrañaga y Yubero, 2015; Ortega et al., 2016). Además, la cibervíctima desconfía de los profesores y de la autoridad institucional (Buelga, Cava y Torralba, 2014), por lo que no pide ayuda para afrontar la situación. Y a lo anterior habría que añadir una cosa más: la exigencia por convertirse en un "adulto" autosuficiente a nivel universitario, lo que agrava su aislamiento y vulnerabilidad.

Teniendo como marco las anteriores ideas y partiendo de que el fenómeno del ciberacoso ha sido menos estudiado en muestras de jóvenes universitarios (Resett y Putallaz, 2018), el presente trabajo de investigación se planteó los siguientes objetivos: 1) examinar en qué medida se relaciona la cibervictimización con las variables individuales (aceptación percibida y depresión) y escolares (implicación, afiliación y ayuda del profesor), y a su vez, describir las diferencias de las variables en función del sexo; 2) analizar las posibles divergencias entre los grupos (ocasionales, severos y sin cibervictimización) y las variables individuales y escolares, y 3 ) puntualizar el valor predictivo de las variables individuales y escolares en la cibervictimización. 


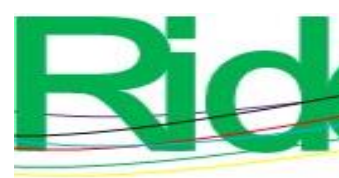

Revista Iberoamericana para la Investigación y el Desarrollo Educativo ISSN $2007-7467$

\section{Método}

\section{Participantes}

Este estudio fue de tipo explicativo y se utilizó un diseño transversal. La muestra estuvo conformada por 662 jóvenes de segundo semestre de las diferentes carreras del Centro Universitario de la Costa de la Universidad de Guadalajara, ubicado en Puerto Vallarta, Jalisco, México; fue, además, representativa de las tres divisiones: Ciencias Biológicas y de la Salud, Estudios Sociales y Económicos e Ingenierías. En este estudio participaron 282 hombres (42.5\%) y 380 mujeres (57.5\%), el rango de edad comprendió entre los 18 y 25 años, con la media total de 19.41 años $(\mathrm{DT}=2.43)$.

\section{Procedimiento}

Se solicitó la autorización a las autoridades correspondientes; se informó a los coordinadores de carrera sobre los objetivos del estudio. En cuanto a los valores éticos en la investigación con seres humanos, el estudio respetó los principios fundamentales incluidos en la Declaración Helsinki, en sus actualizaciones y en la normatividad vigente, tales como consentimiento informado y derecho a la información, protección de datos personales y garantías de confidencialidad, no discriminación, gratuidad y posibilidad de abandonar el estudio en cualquiera de sus fases. Los casos atípicos se atendieron con la detección o presencia de casos atípicos univariantes y multivariantes. Los primeros se detectaron mediante la exploración de puntuaciones estandarizadas. Siguiendo los criterios indicados por Hair, Anderson, Tathan y Black (2008), se consideraron valores atípicos aquellos cuyas puntuaciones estandarizadas presentaran un valor absoluto superior a cuatro. Los segundos se detectaron al computar la distancia de Mahalanobis (Tabachnick y Fidell, 2006).

\section{Instrumentos de evaluación}

En los párrafos siguientes se describen las escalas utilizadas en el presente estudio.

Respecto a la Escala de Victimización a través del Teléfono Móvil y de Internet [CYBVIC] (Buelga et al., 2012), de las dos dimensiones fue utilizada solo la cibervictimización en Internet: la subescala está compuesta por 10 ítems (por ejemplo: "Han contado mentiras o rumores falsos sobre mí"). Los rangos de respuesta de la escala son de uno a cuatro (nunca, algunas veces, 


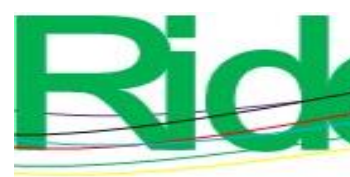

Revista Iberoamericana para la Investigación y el Desarrollo Educativo

ISSN 2007 - 7467

bastantes veces y muchas veces). En el estudio, el coeficiente de fiabilidad (alfa de Cronbach) fue de 0.85 .

La Escala de Aceptación Percibida [PAS] (Brock, Sarason, Sanghvi y Gurung, 1998), adaptada al castellano por Rodríguez, Martínez, Tinajero, Guisande y Páramo (2012), fue utilizada para medir el nivel de las diferentes fuentes de apoyo social. Está compuesta por 44 reactivos agrupados en cuatro dimensiones: 1) PAS-Amigos, conformada por 12 ítems (por ejemplo: "Soy importante para mis amigos"); 2) PAS-Familia, integrada por 12 ítems (p. ej.: "Mis padres se oponen a algunas cosas que hago"); 3) PAS-Madre, formada por 10 ítems (p. ej.: "Mi madre siempre está disponible cuando la necesito"), y 4) PAS-Padre, compuesta por 10 ítems (p. ej.: "Mi padre nunca me ha comprendido"). Los rangos de respuesta son del uno al cinco (total desacuerdo, en desacuerdo, ni de acuerdo ni desacuerdo, de acuerdo, totalmente de acuerdo). El coeficiente de fiabilidad $\alpha$ obtenido para este estudio fue de $0.84,0.89,0.85$ y 0.86 , respectivamente. En la escala global se reportó un alfa de 0.93 .

La Escala de Clima Escolar [CES] (Moos, Moos y Trickett, 1984) fue utilizada para medir el clima social y las relaciones interpersonales existentes en el aula, adaptada al castellano por Fernández y Sierra (1989). La escala está compuesta por 30 ítems agrupados en tres dimensiones: 1) implicación, formada por 10 ítems (p. ej.: "Los alumnos/as ponen mucho interés en lo que hacen en clases”); 2) afiliación, integrada por 10 ítems (p. ej.: "En clases, los alumnos/as llegan a conocerse bien unos a otros"), y 3) ayuda del profesor, formada también por 10 ítems (p. ej.: “Los profesores/as muestran interés personal por los alumnos/as"). Los rangos de respuestas son dos (falso y verdadero). En esta investigación, los coeficientes de fiabilidad (alfa de Cronbach) fueron de 0.68 para implicación, 0.67 para afiliación y 0.61 para ayuda del profesor, y el coeficiente global de la escala fue 0.81 .

La Escala de Depresión [CESD] (Radloff, 1977) fue utilizada para medir la sintomatología asociada a la depresión o al ánimo depresivo. Está compuesta por siete ítems agrupados en una dimensión (p. ej.: "Me sentí deprimido"). Los rangos de respuesta son del uno al cuatro (nunca, pocas veces, muchas veces y siempre). En esta investigación, el coeficiente de fiabilidad (alfa de Cronbach) fue de 0.77 . 


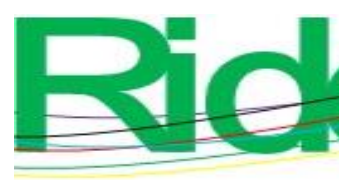

Revista Iberoamericana para la

Investigación y el Desarrollo Educativo

ISSN 2007 - 7467

\section{Resultados}

La codificación y análisis de los datos se realizó en el paquete estadístico SPSS versión 22. Primero se analizaron las correlaciones de Pearson para determinar la relación entre cibervictimización con todas las variables estudiadas, así como la aplicación de la prueba $t$ para comprobar las diferencias por sexo. Posteriormente, para clasificar a los jóvenes, se utilizaron las puntuaciones de la escala de cibervictimización a través de Internet, conformando tres grupos: no cibervíctima, cibervíctima ocasional y cibervíctima severa. Siguiendo a Marini, Dane, Bosacki y Ylc-Cura (2006), los jóvenes que puntúan una desviación típica arriba de la media se ubican en el grupo de cibervíctima severas; los que puntúan uno (nunca) se sitúan en el grupo de no cibervíctima y los que obtienen los puntajes restantes se asignan al grupo de cibervíctima ocasional. Estableciendo los grupos de contraste se calculó el análisis multivariante de la varianza (Manova) y el de la varianza (Anova) para analizar las variables individuales y las variables escolares. Finalmente, se realizó el análisis de regresión lineal para examinar el valor predictivo de las variables referidas con la cibervictimización. En los siguientes párrafos se detallan los datos recabados acorde a los procedimientos estadísticos realizados.

\section{Correlaciones de Pearson}

En la tabla 1 se presentan las correlaciones entre las variables del estudio, las medias, las desviaciones típicas y la prueba $t$ correspondientes. Se obtuvieron correlaciones significativas entre todas las variables. La cibervictimización correlaciona de manera significativa y negativa con aceptación percibida de los amigos $(r=-0.257, p<0.01)$, aceptación percibida de la familia ( $r=-0.278, p<0.01)$, implicación escolar $(r=-0.215, p<0.01)$, afiliación escolar $(r=-0.243, p$ $<0.01)$ y ayuda del profesor $(r=-0.167, p<0.01)$; a su vez, correlaciona positivamente con depresión $(r=0.259, p<0.01)$. 


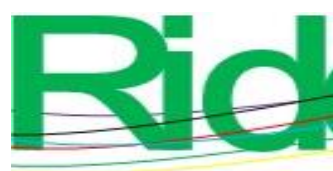

Revista Iberoamericana para la Investigación y el Desarrollo Educativo

ISSN $2007-7467$

Tabla 1. Correlaciones Pearson, medias, desviaciones típicas y pruebas t entre las variables consideradas

\begin{tabular}{|c|c|c|c|c|c|c|c|c|c|}
\hline & 1 & 2 & 3 & 4 & 5 & 6 & 7 & 8 & 9 \\
\hline $\begin{array}{l}\text { 1. Victimización } \\
\text { Internet }\end{array}$ & 1 & & & & & & & & \\
\hline $\begin{array}{l}2 . \\
\text { Sintomatología } \\
\text { depresiva }\end{array}$ & $.259^{* *}$ & 1 & & & & & & & \\
\hline 3. PAS Amigos & $-.257^{* *}$ & $-.399^{* *}$ & 1 & & & & & & \\
\hline 4. PAS Familia & $-.278^{* *}$ & $-.479^{* *}$ & $.470^{* *}$ & 1 & & & & & \\
\hline 5. PAS Madre & $-.255^{* *}$ & $-.340^{* *}$ & $.315^{* *}$ & $.764^{* *}$ & 1 & & & & \\
\hline 6. PAS Padre & $-.230^{* *}$ & $-.338^{* * *}$ & $.309^{* *}$ & $.634^{* *}$ & $.510^{* *}$ & 1 & & & \\
\hline 7. Implicación & $-.215^{* *}$ & $-.296^{* *}$ & $.261^{* *}$ & $.262^{* *}$ & $.221^{* *}$ & $\begin{array}{c}.261^{*} \\
*\end{array}$ & 1 & & \\
\hline 8. Afiliación & $-.243^{* *}$ & $-.233^{* *}$ & $.287^{* *}$ & $.258^{* *}$ & $.222^{* *}$ & $\begin{array}{c}.256^{*} \\
*\end{array}$ & $.486^{* *}$ & 1 & \\
\hline $\begin{array}{l}\text { 9. Ayuda del } \\
\text { profesor }\end{array}$ & $-.167^{* *}$ & $-.177^{* *}$ & $.220^{* *}$ & $.207^{* *}$ & $.222^{* *}$ & $\begin{array}{c}.139^{*} \\
*\end{array}$ & $.440^{* *}$ & $.369^{* *}$ & 1 \\
\hline M Hombre & 1.44 & 1.96 & 3.60 & 4.04 & 4.17 & 3.86 & 1.58 & 1.69 & 1.64 \\
\hline DT Hombres & .54 & .50 & .63 & .65 & .61 & .77 & .23 & .21 & .21 \\
\hline M Mujer & 1.38 & 2.15 & 3.61 & 3.83 & 4.00 & 3.63 & 1.54 & 1.68 & 1.59 \\
\hline DT Mujeres & .48 & .50 & .65 & .77 & .76 & .87 & .23 & .23 & .22 \\
\hline $\mathrm{T}$ & 1.35 & $-4.79^{* * *}$ & -.20 & $\begin{array}{c}3.73^{* *} \\
*\end{array}$ & $3.4^{* *}$ & $\begin{array}{c}3.58^{*} \\
* *\end{array}$ & $2.70^{* *}$ & .54 & $\begin{array}{c}3.25 \\
* *\end{array}$ \\
\hline
\end{tabular}

Nota: $M=$ Medias H/M; $D E=$ Desviación estándar; $T=$ Prueba $t$ de Student.

$* \mathrm{p}<0,05 ; * * \mathrm{p}<0,01 ; * * * \mathrm{p}<0,001$.

Fuente: Elaboración propia

También se encontraron diferencias estadísticamente significativas entre los sexos en las variables de sintomatología depresiva $(t=-4.79, p<0.001)$, aceptación percibida de la familia $(t$ $=3.73, p<0.001)$, aceptación percibida de la madre $(t=3.14, p<0.01)$, aceptación percibida del padre $(t=3.58, p<0.001)$, implicación escolar $(t=2.70, p<0.01)$ y ayuda del profesor $(t=3.25$, $p<0.01)$. Los chicos puntúan medias más altas en aceptación percibida de la familia, aceptación 


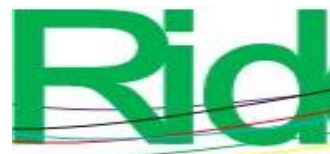

.
Revista Iberoamericana para la Investigación y el Desarrollo Educativo

ISSN $2007-7467$

percibida de la madre, aceptación percibida del padre, implicación escolar y ayuda del profesor; en cambio, las chicas sobresalen en sintomatología depresiva.

\section{Manova y Anova de los grupos de cibervíctima y las variables individuales y escolares}

Posteriormente se procedió a realizar el Anova, el cual reveló diferencias estadísticamente significativas entre los grupos de cibervíctima con las variables individuales y escolares $(\wedge=0.992$, $\left.F(16,1306)=5.48, p<0.001, n^{2}=0.063\right)$.

El Anova mostró diferencias significativas en sintomatología depresiva $(F,(2.659)=21.69$, $\left.P<0.001, n^{2}=0.072\right)$, aceptación percibida de los amigos $\left(F,(2.659)=15.86, P<.001, \mathrm{n}^{2}=.046\right)$, aceptación percibida de la familia $\left(F,(2.659)=25.48, P<0.001, n^{2}=0.072\right)$, aceptación percibida de la madre $\left(F,(2.659)=17.07, P<0.001, n^{2}=0.049\right)$, aceptación percibida del padre $(F,(2.659)$ $\left.=16.34, P<0.001, n^{2}=0.047\right)$, implicación $\left(F,(2.659)=10.47, P<0.001, n^{2}=0.032\right)$, afiliación $\left(\mathrm{F},(2.659)=15.34, P<0.001, n^{2}=0.044\right)$ y ayuda del profesor $\left(F,(2.659)=7.65, P<0.001, n^{2}\right.$ $=0.017)$.

Así, como se constata en la tabla 2, las pruebas Bonferroni indicaron que los jóvenes no cibervíctima obtuvieron las puntuaciones estadísticamente más elevadas en aceptación percibida de los amigos, aceptación percibida de la familia, aceptación percibida de la madre, aceptación percibida del padre y afiliación escolar, diferenciándose de los jóvenes con moderada y severa cibervictimización. En la variable de implicación escolar y ayuda del profesor no hay diferencias en las medias de los grupos de no cibervíctima y ocasionales cibervíctima, pero sí existen discrepancias entre ambos grupos con respecto a las víctimas severa. Además, los jóvenes con severa cibervictimización mostraron una mayor sintomatología depresiva respecto a los colectivos de moderada y no cibervictimización. 


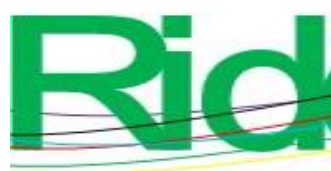

Revista Iberoamericana para la Investigación y el Desarrollo Educativo

ISSN $2007-7467$

Tabla 2. Diferencias entre los grupos (no cibervíctimas, cibervíctimas ocasionales y cibervíctimas severas) en las variables individuales y escolares

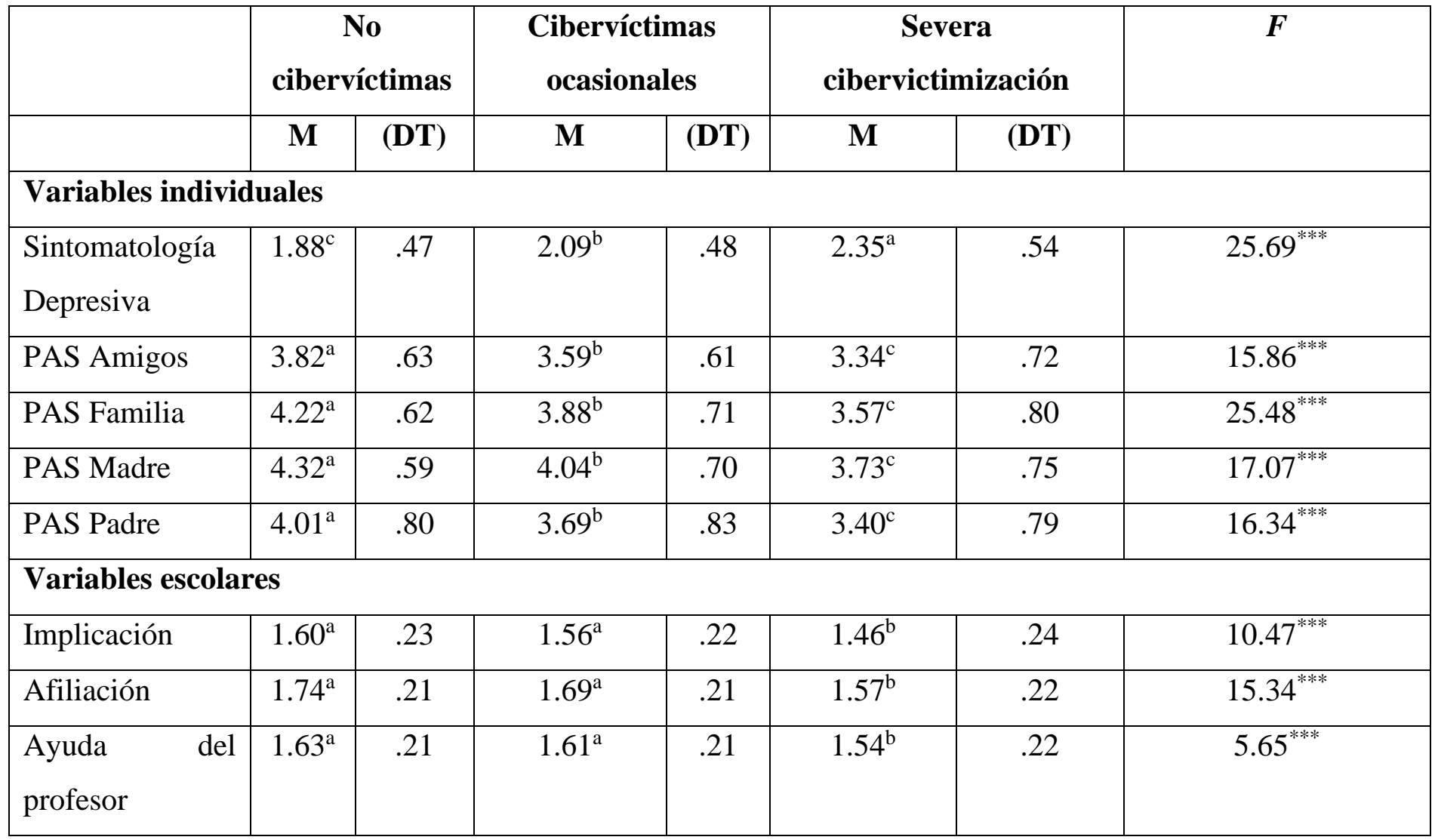

Nota: $M=$ Media; $D T=$ Desviación típica; $F=\mathrm{F}$ de Fisher-Snedecor; ${ }^{\mathrm{F}}$ Prueba de Bonferroni. $A>b>c$

$* p<0,05 ; * * p<0,01 ; * * * p<0,001$.

Fuente: Elaboración propia

\section{Valor predictivo de las variables individuales y escolares en la cibervictimización}

Por último, los resultados del análisis de regresión confirmaron el valor predictivo de las variables individuales y escolares en la cibervictimización. Como se verifica en la tabla 3, por un lado, las variables individuales explican $11.4 \% \mathrm{y}$, por el otro, las variables escolares $7.0 \%$ de la cibervictimización. Es de subrayar que las variables individuales poseen un valor predictivo más alto que las variables escolares.

Se constata que la aceptación percibida de los amigos $(\beta=-0.139 ; p=<0.001)$, la sintomatología depresiva $(\beta=0.135 ; p=<0.002)$ y la aceptación percibida de la madre $(\beta=0.119$; $p=<0.038$ ) son variables explicativas estadísticamente significativas; a su vez, los factores de 


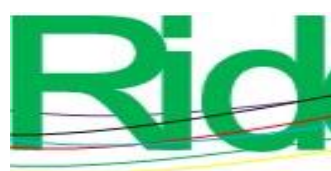

Revista Iberoamericana para la Investigación y el Desarrollo Educativo

ISSN 2007 - 7467

aceptación percibida de la familia y aceptación percibida del padre no son significativas en la dimensión predictiva de la cibervictimización.

En cuanto a las variables escolares, se constata que la implicación escolar $(\beta=-0.108 ; p$ $=<0.017)$ y la afiliación $(\beta=0.169 ; p=<0.001)$ son variables estadísticamente significativas en la explicación de la cibervictimización; en cambio, la variable ayuda del profesor no es significativa.

Tabla 3. Variables predictoras de cibervictimización

\begin{tabular}{|l|c|c|c|c|}
\hline \multicolumn{1}{|c|}{ Variables predictoras } & $\begin{array}{c}\boldsymbol{R}^{\mathbf{2}} \\
\text { corregida }\end{array}$ & $\boldsymbol{F}$ & $\boldsymbol{\beta}$ & \\
\hline Variables individuales & .114 & 17.95 & & \\
\hline Sintomatología Depresiva & & & -.135 & $.002^{*}$ \\
\hline PAS Amigos & & & -.139 & $.001^{*}$ \\
\hline PAS Familia & & & -.015 & .831 \\
\hline PAS Madre & & & -.119 & $.038^{*}$ \\
\hline PAS Padre & & & -.066 & .165 \\
\hline Variables escolares & .070 & 17.61 & & $.017^{*}$ \\
\hline Implicación & & & -.108 & $.000^{*}$ \\
\hline Afiliación & & & -.169 & .181 \\
\hline Ayuda del profesor & & & -.057 & \\
\hline
\end{tabular}

Nota: $R^{2}=$ Correlación múltiple cuadrada; $F=F$ de Fisher-Snedecor; $\beta=$ Beta; $p=\alpha=0.05$.

Fuente: Elaboración propia

\section{Discusión}

En la presente investigación se planteó el objetivo de analizar las relaciones entre las variables individuales y escolares y su relación con la cibervictimización.

Previo al propósito del estudio, los resultados descriptivos revelaron que $23.41 \%(n=155)$ de los jóvenes nunca han sido victimizados por Internet, $63.74 \%(n=422)$ ha sido de forma ocasional y $12.83 \%$ de forma severa $(n=85)$. Este último dato coincide con los estudios de Garaigordobil (2015), quien reporta una cifra de $15.8 \%$ de cibervíctimas severas. A la vez, los resultados confirman que hay diferencias significativas en cuanto al sexo en las variables de 


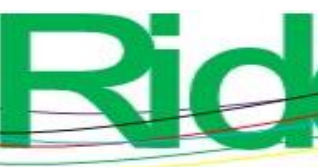

Revista Iberoamericana para la Investigación y el Desarrollo Educativo

ISSN 2007 - 7467

aceptación percibida de la familia, aceptación percibida de la madre, aceptación percibida del padre, implicación escolar y ayuda del profesor; destacan en sus medias los varones sobre las mujeres. En los hombres, a diferencia de las mujeres, se acentúan más recursos psicológicos de su familia y de su red social, al sentirse aceptados y reconocidos en sus vínculos relacionales, lo cual se traslada en la universidad al implicarse con sus compañeros y al solicitar ayuda de los profesores. Por otro lado, las mujeres sobresalen en la media de sintomatología depresiva, situación que las ubica en una vulnerabilidad psicológica que podría facilitar la situación de verse involucrada en el fenómeno del acoso cibernético, en el papel de cibervíctima. Este resultado es coincidente con otros estudios al señalar que las mujeres suelen ser más cibervictimizadas que los hombres (Ortega et al., 2017; Resett y Putallaz, 2018).

Los hallazgos permiten confirmar que la cibervictimización se relaciona significativamente con las variables individuales y escolares estudiadas. Los datos indican que los jóvenes no cibervictimizados, en comparación con los ocasionales y severos, presentan mejor ajuste en las variables individuales y escolares. Así, los jóvenes no cibervictimizados tienen una mejor aceptación percibida de los amigos, aceptación percibida de la familia, aceptación percibida de la madre y aceptación percibida del padre. En el sentido de que la red de coetáneos, familia, la madre y el padre, lo aceptan, lo acogen emocionalmente, lo respetan en sus decisiones, son sensibles a sus necesidades, cuenta con ellos para contarle secretos, en suma, un tejido colectivo de personajes que le genera un clima positivo y un recurso más para reafirmar su identidad social, su proyecto de vida, los sentimientos de ser querido, reconocido y amado. En este proceso de aceptación se encuentra articulada de forma implícita una comunicación abierta en donde se transmiten los valores de respeto, aceptación, sentimientos positivos con una calidez emocional. Estos resultados son coincidentes con otros estudios, pues señalan que existe una relación entre la comunicación familiar abierta y el cyberbullying (Law, Shapka y Olson, 2010), ya que una paternidad sensible, cálida y afectuosa utiliza la comunicación abierta entre los integrantes de la familia y establece valores de respeto entre los jóvenes, y por ende, los aleja de las agresiones en el mundo virtual (Yubero, Larrañaga y Navarro, 2014). Es importante señalar que la aceptación percibida parte de vivenciar una parentalidad afectuosa y un estilo de crianza con normas, integrando los elementos protectores en la familia que disminuyen la posibilidad de incurrir en los fenómenos desadaptativos como el ciberacoso (Hinduja y Patchin, 2013; Ortega et al., 2016; Sasson y Mesch, 2014). 


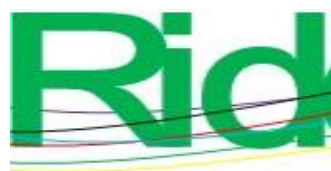

Revista Iberoamericana para la Investigación y el Desarrollo Educativo

ISSN 2007 - 7467

Otra variable en la cual destaca el grupo de no cibervictimizados en comparación a los ocasionales y severos es la afiliación escolar. El joven que no vive la agresión por Internet interactúa en un clima escolar positivo caracterizado por buenas relaciones en el grupo, un conocimiento mutuo de los compañeros, un sentimiento solidario, trabajo colectivo y amistad en el ámbito universitario; elementos que propician una retroalimentación positiva en su identidad en su bienestar psicológico y confluye con la percepción de la aceptación de los padres, la familia y amigos. Los datos arrojados son coincidentes con otras investigaciones al señalar que los jóvenes no cibervictimizados presentan una alta autoestima académica y familiar (Tokunaga, 2010), así como la identificación con sus pares (Buelga y Pons, 2012; Tokunaga, 2010) y relaciones positivas con sus compañeros de clase (Odaci y Kalhan, 2010).

Otro dato particular de la investigación es que el grupo de ocasionales y no cibervictimizados destacaron en sus medias en implicación escolar y ayuda del profesor en contraste con el grupo de severa cibervictimización. Este resultado puede entenderse en el contexto de que los jóvenes son mayores de edad y socialmente se les exige una autonomía responsable, lo cual se implica en las actividades en clase, poniendo atención, participando activamente, haciendo trabajos, con una figura de autoridad del profesorado no tan potente como en los anteriores niveles educativos, acompañado de una exigencia y de responsabilidad como adulto emergente. Como plantea Ortega y Torralba (2017), es importante que los alumnos presenten un respeto hacia las figuras de autoridad e instituciones formales; acompañado por la confianza con los profesores y una apreciación positiva de la escuela como elementos protectores en un clima escolar que mitigue los problemas de cibervictimización y ciberacoso (Buelga et al., 2014).

Los jóvenes cibervíctimas severos muestran peor ajuste en las variables individuales y escolares que los grupos de cibervíctimas ocasionales y no cibervíctimas. Así, en el ámbito individual, los resultados indican que los jóvenes cibervictimizados de forma severa tienen una sintomatología depresiva, con problemas de sueño, falta de esfuerzo y dificultad para concentrarse, situación que propicia un malestar subjetivo que mantiene una percepción negativa de los amigos, familia, específicamente de la madre y el padre. Los resultados son coincidentes con otras investigaciones que plantean que las víctimas severas de ciberacoso presentan depresión (Ortega et al., 2017; Resett y Putallaz, 2018; Roberts, Axas, Nesdole y Repetti, 2016), un elevado malestar psicológico, ideación suicida (Avilés, 2013), baja autoestima (Ortega et al., 2016; Tokunaga, 2010), todo lo cual afecta su salud mental y reduce los recursos personales y sociales (Makri y Karagianni, 2014; Ortega et al., 2016). 


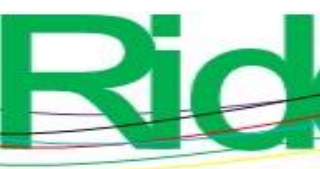

Revista Iberoamericana para la Investigación y el Desarrollo Educativo

ISSN 2007 - 7467

Las cibervíctimas severas son las que presentan la peor aceptación percibida de la red de amigos, familia, madre y padre, así como un clima escolar negativo en las áreas de implicación, afiliación y ayuda del profesor, que en suma propicia una constelación sistémica de vulnerabilidad al carecer de recursos individuales y escolares. Los resultados presentan similitudes con otros estudios cuyos hallazgos confirman que las víctimas de ciberacoso mantienen una escasa comunicación familiar (Larrañaga et al., 2016) o vivencian una comunicación ofensiva y evitativa (Yubero et al., 2014) que descalifica su identidad, lo que las hace entrar en una circularidad de conflictos familiares (Ortega et al., 2016). La vulnerabilidad subjetiva que vive la cibervíctima propicia la desconfianza hacia los profesores, que impide el solicitar ayuda para detener la situación de maltrato (Ortega, Buelga, Cava y Torralba, 2017) y sobre todo por la idea de que es adulto y tiene que defenderse en el ámbito universitario, aunado a una percepción de menor ayuda y amistad con los compañeros (Ortega et al., 2016). También padecen una sensación de baja afiliación entre pares, lo que afecta su participación en tareas e impacta negativamente en el rendimiento escolar (Buelga et al., 2014).

Finalmente, este trabajo proporciona observaciones sugerentes y relevantes sobre ciertas variables psicosociales que intervienen en el fenómeno del ciberacoso en jóvenes universitarios; contiene las fortalezas de ser de tipo correlacional y explicativo con una muestra representativa en una población poco estudiada como son los jóvenes universitarios; asimismo, se utilizaron escalas estandarizadas en México, lo que permitió un análisis de datos multivariados y de regresión. Sin embargo, es importante reseñar las limitaciones del estudio. Los resultados expuestos deben interpretarse con cautela debido a la naturaleza transversal y correlacional de los datos que, como es bien sabido, no permite establecer relaciones causales entre las variables. Un estudio longitudinal con medidas en distintos tiempos ayudaría a la clarificación de las relaciones aquí observadas. Otra salvedad es que la utilización de escalas en la modalidad autoinforme puede generar sesgos y deseabilidad social al ser contestada por el joven, por lo que se recomienda en otros estudios incluir la percepción de los compañeros de clase y los profesores. Una limitante más para la generalización de resultados a nivel universitario es que esta investigación conformó su universo únicamente con los estudiantes de segundo semestre; una muestra estratificada con todos los semestres de las carreras acompañada del incremento en su tamaño ayudaría a redimir lo anterior. Pese a estas limitaciones, este trabajo puede efectivamente orientar a quienes diseñan programas de prevención e intervención en el ámbito del ciberacoso en universitarios, integrando a la familia y la escuela en la atención y explicación del mismo. 


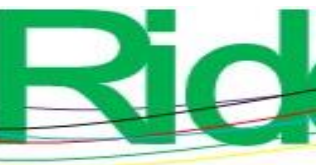

Revista Iberoamericana para la Investigación y el Desarrollo Educativo

ISSN 2007 - 7467

Los resultados señalados orientan sobre la permanencia de programas de tutorías a nivel universitario, y revelan que, a pesar de encontrarse en la etapa de "adultez emergente", los jóvenes se implican en situaciones de acoso en Internet y los más vulnerables son las víctimas. El fenómeno debe abordarse sistemáticamente, atendiendo temas en la formación integral que propicien una individuación con recursos protectores en las áreas de salud mental, ampliando los vínculos sociales con amigos, padres, compañeros de clase y profesores, los cuales retroalimentan la percepción de su personalidad y su trascendencia relacional en la vida escolar y social. Un eje de la operatividad del programa sería sensibilizar a los jóvenes acerca de la convivencia pacífica, empática, así como activar a compañeros mediadores en la solución de conflictos (Buelga et al., 2014; Ortega et al., 2017). Otro eje sería la intervención psicoeducativa para reducir las colisiones de los implicados y el apoyo terapéutico a las víctimas y a los agresores (Garaigordobil, 2015).

\section{Conclusiones}

El ciberacoso es un fenómeno que se presenta en el ámbito universitario, en donde se maltrata e insulta utilizando el Internet con la intención de repetir conductas agresivas para dañar o molestar a la víctima. La cibervictimización se relaciona significativamente con las variables individuales y escolares. El peor ajuste psicológico lo vivencia la cibervíctima severa, al percibir la pésima aceptación de la madre, el padre, la familia y amigos. A su vez, destacan con una mayor sintomatología depresiva, que se articula con un clima escolar negativo caracterizado por poca cohesión grupal y apoyo de los profesores, situación que los ubica en una constelación de vulnerabilidad psicológica en su ser, en las redes sociales y escolares de convivencia. En cambio, los no implicados en la cibervictimización presentan un mejor ajuste psicológico en las variables individuales y escolares; la aceptación percibida de los amigos, familia y padres es positiva y tienen menor sintomatología depresiva. Todos ellos son elementos individuales que nutren positivamente su identidad, sus habilidades y recursos para convivir con sus compañeros de clase, implicándose en un clima escolar positivo. Finalmente, en la explicación de la cibervictimización destacan las variables individuales sobre las variables escolares. 


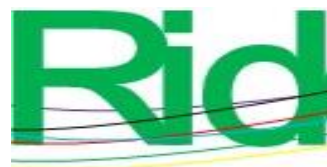

\section{Referencias}

Arab, L. E. y Díaz, G. A. (2015). Impacto de las redes sociales e internet en la adolescencia: aspectos positivos y negativos. Revista Médica Clínica Las Condes, 26(1), 7-13.

Avilés, J. M. (2013). Análisis psicosocial del ciberbullying: claves para una educación moral. Papeles del Psicólogo, 34(1), 65-73. Recuperado de http://www.papelesdelpsicologo.es/pdf/2172.pdf.

Brock, D. M., Sarason, I. G., Sanghvi, H. and Gurung, R. A. R. (1998). The Perceived Acceptance Scale: Development and validation. Journal of Social and Personal Relationships, 15(1), 521. Retrieved from https://doi.org/10.1177/0265407598151001.

Buelga, S. y Chóliz, M. (2013). El adolescente frente a las nuevas tecnologías de la información y la comunicación. En Musitu, G. (ed.), Adolescencia y familia: Nuevos retos en el siglo XXI (pp. 209-228). Ciudad de México, México: Editorial Trillas.

Buelga, S. y Pons, J. (2012). Agresiones entre adolescentes a través del teléfono móvil y de internet. Psychosocial Intervention, 21(1), 91-101.

Buelga, S., Cava, M. J. y Musitu, G. (2012). Validación de la Escala de Victimización entre Adolescentes a través del Teléfono Móvil y de Internet. Pan American Journal of Public Health, 32(1), 36-42. Recuperado de https://doi.org/10.1590/S1020-49892012000700006.

Buelga, S., Cava, M. J. y Torralba, E. (2014). Influence of family environment in victims of cyberbullying. Paper presented at the XII National Congress of Social Psychology. Sevilla, November 2014.

Buelga, S., Martínez, B. y Musitu, G. (2016). Family relationships and cyberbullying. In Navarro, R., Yubero, S. and Larrañaga (eds.), Cyberbullying Across the Globe (pp. 99-114). New York, United States: Springer International Publishing.

Cappadocia, M. C., Craig, W. M. and Pepler, D. (2013). Cyberbullying: prevalence, stability, and risk factors during adolescence. Canadian Journal of School Psychology, 28(2), 171-192.

Cross, D., Barnes, A., Papageorgiou, A., Hadwen, K., Hearn, L. and Lester, L. (2015). A socialecological framework for understanding and reducing cyberbullying behaviours. Aggression and Violent Behavior, 23, 109-117.

Durán, M. y Martínez, R. (2015). Ciberacoso mediante teléfono móvil e Internet en las relaciones de noviazgo entre jóvenes. Comunicar, 22(44). Recuperado de http://dx.doi.org/10.3916/C44-2015-17. 


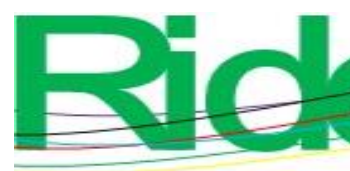

Revista Iberoamericana para la Investigación y el Desarrollo Educativo

ISSN 2007 - 7467

Fernández, R. y Sierra, B. (1989). Escalas de clima social: FES, WES, CIES y CES. Madrid, España: TEA.

Gálvez, J. L., Vera, D., Cerda, C. y Díaz, R. (2016). Escala de Victimización entre Adolescentes a través del Teléfono Móvil y de Internet: Estudio de validación de una versión abreviada en estudiantes chilenos. Revista Iberoamericana de Diagnóstico $e$ Avaliação Psicológica, 41(1), 16-27.

Garaigordobil, M. and Martínez, V. (2016). Impact of Cyberprogram 2.0 on different types of school violence and aggressiveness. Frontiers in Psychology, 7, 428.

Giménez, A. M., Maquilón, J. J. y Sánchez, P. A. (2014). Acceso a las tecnologías, rendimiento académico y cyberbullying en escolares de secundaria. Revista Iberoamericana de Psicología y Salud, 5(2), 119-133.

Gualdo, A. M., Hunter, S. C., Durkin, K., Arnaiz, P. and Maquilón, J. J. (2015). The emotional impact of cyberbullying: Differences in perceptions and experiences as a function of role. Computers \& Education, 82, 228-235.

Hair, J., Anderson, R., Tatham, R. and Black, W. (2008). Multivariate Data Analysis. Boston, United States: McGraw-Hill.

Hinduja, S. and Patchin, J. W. (2013). Social influences on cyberbullying behaviors among middle and high school students. Journal of Youth and Adolescence, 42(5), 711-722.

Kowalski, R. and Limber, S. (2007). Electronic bullying among middle school students. Journal of Adolescent Health, 41(6), S22-S30. $\quad$ Retrieved from http://dx.doi.org/10.1016/j.jadohealth.2007.08.017.

Kowalski, R., Giumetti, G., Schroeder, A. and Lattanner, M. (2014). Bullying in the Digital Age: A Critical Review and Meta-analysis of Cyberbullying Research among Youth. Psychological Bulletin, 140(4), 1073-1137. Retrieved from http://dx.doi.org/10.1037/a0035618 .

Kowalski, R., Limber, S. y Agatston, P. (2010). Cyberbullying: El acoso escolar en la era digital. Bilbao,España: Desclée de Brouwer.

Kowalski, R., Morgan, C. A. and Limber, S. P. (2012). Traditional bullying as a potential warning sign of cyberbullying. School Psychology International, 33(5), 505-519. Retrieved from https://doi.org/10.1177/0143034312445244. 


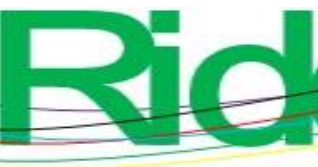

Revista Iberoamericana para la Investigación y el Desarrollo Educativo ISSN 2007 - 7467

Larrañaga, E., Yubero, S., Ovejero, A. and Navarro, R. (2016). Loneliness, parent-child communication and cyberbullying victimization among Spanish youths. Computers in Human Behavior, 65, 1-8.

Law, D. M., Shapka, J. D. and Olson, B. F. (2010). To control or not to control? Parenting behaviours and adolescent online aggression. Computers in Human Behavior, 26(6), 1651-1656.

Lozano, L. M., Valor, I., Pedrosa, I., Suárez, J., García, E. y Lozano L. (2016). Adaptación del Inventario de la Triada Cognitiva Infantil en población española. Anales de Psicología, 32(1), 158-166.

Lucas, B., Pérez, A. y Giménez, M. (2016). La evaluación del cyberbullying: situación actual y retos futuros. Papeles del Psicólogo, 37(1), 27-35

Makri, E. and Karagianni, G. (2014). Cyberbullying in Greek adolescents: The role of parents. Procedia-Social and Behavioral Sciences, 116, 3241-3253.

Marini, Z. A., Dane, A. V., Bosacki, S. L. and Ylc-Cura. (2006). Direct and Indirect Bully-Victims: Differential Psychosocial Risk Factors Associated With Adolescents Involved in Bullying and Victimization. Aggressive Behavior, 32(6), 551-569. Retrieved from https://doi.org/10.1002/ab.20155.

Martínez, I., Murgui, S., García, O. F. and García F. (2019). Parenting in the digital era: Protective and risk parenting styles for traditional bullying and cyberbullying victimization. Computers in Human Behavior, 90, 84-92. Retrieved from https://doi.org/10.1016/j.chb.2018.08.036.

Monelos, M. E., Mendiri, P. y García, C. D. (2015). El bullying revisión teórica, instrumentos y programas de intervención. Revista de Estudios e Investigación en Psicología y Educación, (2), 74-78. Recuperado de https://doi.org/10.17979/reipe.2015.0.02.1299.

Moos, R., Moos, B. y Trickett, E. (1984). FES, WES y CES: Escalas de clima social. Madrid España: TEA Ediciones.

Navarro, R., Ruiz, R., Larrañaga, E. and Yubero, S. (2015). The impact of cyberbullying and social bullying on optimism, global and school-related happiness and life satisfaction among 1012-year-old schoolchildren. Applied Research in Quality of Life, 10(1), 15-36.

Odaci, H., \& Kalkan, M. (2010). Problematic Internet Use, Loneliness and Dating Anxiety among Young Adult University Students. Computers \& Education, 55(3), 1091-1097. Retrieved from https://doi.org/10.1016/j.compedu.2010.05.006. 


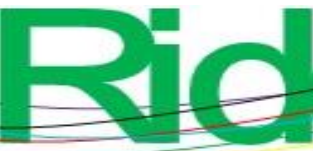

Ortega, J., Buelga, S. y Cava, M. J. (2016). Influencia del clima escolar y familiar en adolescentes víctimas de ciberacoso. Comunicar, 24(46), 57-65. Recuperado de http://dx.doi.org/10.3916/C46-2016-06.

Ortega, J., Buelga, S., Cava, M. J. y Torralba, E. (2017). Violencia escolar y actitud hacia la autoridad de estudiantes agresores de cyberbullying. Revista de Psicodidáctica, 22(1), 2328.

Ortega, J., Torralba, E. y Buelga, S. (2017). Distrés psicológico en adolescentes víctimas de cyberbullying. Revista de Estudios e Investigación en Psicología y Educación, 4(1), 10-17. Recuperado de https://doi.org/10.17979/reipe.2016.4.1.1767.

Patchin, J. W. and Hinduja, S. (2015). Measuring cyberbullying: Implications for research. Aggression and Violent Behaviour, 23, 69-74. Retrieved from https://doi.org/10.1016/j.avb.2015.05.013.

Radloff, L. S. (1977). The CES-D Scale: A Self-Report Depression Scale for Research in the General Population. Applied Psychological Measurement, 1(3), 385-401. Retrieved from https://doi.org/10.1177/014662167700100306.

Resett, S. y Putallaz, P. R. (2018). Cybervictimización y cyberagresión en estudiantes universitarios: problemas emocionales y uso problemático de nuevas tecnologías. Psicodebate, 18(2), 38-50. $\quad$ Recuperado de https://doi.org/10.18682/pd.v18i2.811.

Roberts, N., Axas, N., Nesdole, R. and Repetti, L. (2016). Pediatric emergency department visits for mental health crisis: Prevalence of cyber-bullying in suicidal youth. Child and Adolescent Social Work Journal, 33(5), 469-472. Retrieved from https://doi.org/10.1007/s10560-0160442-8.

Rodríguez, M. S., Martínez, Z., Tinajero, C., Guisande, M. A. y Páramo, M.F. (2012). Adaptación española de la Escala de Aceptación Percibida (PAS) en estudiantes universitarios. Psicothema, 24, 483-488.

Romero, A., Martínez, B., Musitu, G., León, C., Villarreal, M. E. and Callejas, J. E. (2019). Family communication problems, psychosocial adjustment and cyberbullying. International Journal of Environmental Research and Public Health, 16(13), 2417. Retrieved from https://doi.org/10.3390/ijerph16132417.

Sasson, H. and Mesch, G. (2014). Parental mediation, peer norms and risky online behavior among adolescents. Computers in Human Behavior, 33, 32-38. 


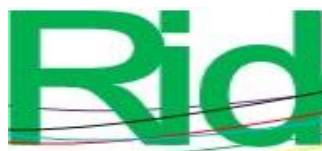

Revista Iberoamericana para la Investigación y el Desarrollo Educativo

ISSN $2007-7467$

Solecki, S., McLaughlin, K. and Goldschmidt, K. (2014). Promoting positive offline relationships to reduce negative online experiences. Journal of Pediatric Nursing, 29(5), 482-484.

Stewart, R. W., Drescher, C. F., Maack, D. J., Ebesutani, C. and Young, J. (2014). The development and psychometric investigation of the Cyberbullying Scale. Journal of Interpersonal Violence, 29(12), 2218-2238.

Tabachnick, B. and Fidell, L. (2006). Using Multivariate Statistics (5 ${ }^{\text {th }}$ ed.). United States: Allyn \& Bacon/Pearson Education.

Tanrikulu, I. and Campbell, M. (2015). Correlates of traditional bullying and cyberbullying perpetration among Australian students. Children and Youth Services Review, 55, 138-146.

Tokunaga, R. S. (2010). Following you home from school: A critical review and synthesis of research on cyberbullying victimization. Computers in human behavior, 26(3), 277-287.

Trickett, E. J., Leone, P. E., Fink, C. M. and Braaten, S. L. (1993). The perceived environment of special education classrooms for adolescents: A revision of the Classroom Environment Scale. Exceptional Children, 59, 411-420.

Udris, R. (2014). Cyberbullying among high school students in Japan: Development and validation of the Online Disinhibition Scale. Computers in Human Behavior, 41, 253-261.

Yubero, S., Larrañaga, E. y Navarro, R. (2014). La comunicación familiar en la victimización del bullying y el cyberbullying. International Journal of Developmental and Educational Psychology, 5(1), 343-350. 


\begin{tabular}{|l|l|}
\hline Rol de Contribución & Autor (es) \\
\hline Conceptualización & Esperanza Vargas Jiménez \\
\hline Metodología & Raúl Medina Centeno \\
\hline Software & Claudia Gregoria Huerta Zúñiga \\
\hline Validación & Remberto Castro Castañeda \\
\hline Análisis Formal & Remberto Castro Castañeda \\
\hline Investigación & Esperanza Vargas Jiménez \\
\hline Recursos & Raquel Domínguez Mora \\
\hline Curación de datos & Claudia Gregoria Huerta Zúñiga \\
\hline $\begin{array}{l}\text { Escritura - Preparación del } \\
\text { borrador original }\end{array}$ & Remberto Castro Castañeda \\
\hline $\begin{array}{l}\text { Escritura - Revisión } \\
\text { edición }\end{array}$ & Raúl Medina Centeno \\
\hline Visualización & Raquel Domínguez Mora \\
\hline Supervisión & Esperanza Vargas Jiménez \\
\hline $\begin{array}{l}\text { Administración } \\
\text { Proyectos }\end{array}$ & Esperanza Vargas Jiménez \\
\hline Adquisición de fondos & No aplica \\
\hline
\end{tabular}

